\title{
FECHA DE C14 DE LA FASE PROTOCOGOTAS I DEL YACIMIENTO DEL CASERÍO DE PERALES DEL RÍo
}

\author{
M. ${ }^{2}$ CONCEPCIÓN BlasCO BOSQUED \\ juana CALLE PARDo \\ M. ${ }^{2}$ LUZ SÁNCHEZ-CAPILLA \\ Departamento de Prehistoria y Arqueologia UAM
}

\section{Resumen}

El trabajo da a conocer la primera datación de C14 para la fase Protocogotas I en el Valle del Tajo, la muestra analizada procede de una de las "fosas" del yacimiento madrileño del Caserío de Perales del Río y estaba asociada a un contexto muy homogéneo que permite adscribirlo, sin dudas, a la fase de formación del Horizonte Cogotas I. La datación coincide plenamente con las que se conocían procedentes de conjuntos del Valle del Duero con características culturales similares a las del Caserio de Perales.. El resultado arroja una fecha de 1406 a.C. y de 1629 cal BC, confirmando los origenes del Horizonte a mediados del II milenio, en paralelo al desarrollo de otros círculos peninsulares del Bronce Pleno

\section{Summary}

This work releases the first date of $\mathrm{Cl} 4$ for the stage Protocogotas I at Tajo's valley the sign analysed comes from one of the "pits" of Caserio de Perales del Río's settlement which is situated in Madrid's province and it was asociated to an homogeneous context that allow without any doubt to include it on the formation's phase of Cogotas I Horizon. The date coincides exactly with those which come from the Duero's valley. The cronology that we obtained as result: 1406 b.c. and 1629 cal B.C. that confirms the Cogotas's horizon origins was in the middle of the second millenium as the development of other peninsular cultural areas of the Middie Bronze Age.

\section{EL ORIGEN DE COGOTAS I EN EL VALIE DEL TAJO: LA AUSENCIA DE DATACIONES}

Todavia hoy la Edad del Bronce en la submeseta sur tiene numerosas cuestiones pendientes de resolver debido a la falta de yacimientos excavados en extensión que 
cuenten además con una monografia amplia y rigurosa. Este hecho no sólo impide definir con nitidez las características peculiares de cada una de las distintas facies culturales (MARTÍNEZ NAVARRETE, M. ${ }^{2}$ I., 1988), sino que también dificulta obtener una visión precisa de la evolución cultural que se produce en la zona a lo largo de buena parte del segundo milenio anterior a nuestra era e, incluso, saber si dicha evolución se produce de manera paralela o asimétrica en las distintas regiones.

Entre las deficiencias existentes se encuentra la escasez de dataciones absolutas que ayuden a vertebrar el devenir de la Edad del Bronce en la zona y a contrastar las secuencias temporales de las diferentes regiones de esta amplia área del interior, así como su relación con otros ámbitos peninsulares. No obstante algunas de las dataciones llevadas a cabo en yacimientos de la Edad del Bronce de esta zona empiezan a mostrar algunos datos de interés los cuales permiten avanzar en los estudios de la Edad del Bronce de la Meseta sur, aunque estos avances se producen con un ritmo que resulta más lento del deseable.

En un reciente trabajo se ha ofrecido una panorámica de las dataciones de $\mathrm{C} 14$ disponibles sobre la Edad del Bronce en el cuadrante suroriental de la submeseta sur, en él se recopilan un total de 97 fechas que proceden de 13 yacimientos (FERNÁNDEZ-POSSE, M. ${ }^{2}$ D.; Gilman, A., y Martín, C., 1996, 127-130), un elenco a todas luces insuficiente si tenemos en cuenta la amplitud del territorio que cubre y la diversidad de yacimientos que abarca y, sobre todo, el desequilibrio en la representación de dichos círculos ya que 7 de las 13 estaciones datadas son motillas o morras a las que pertenecen 67 de las 97 fechas obtenidas. No obstante, este repertorio permite, al menos, confirmar la hipótesis que, hace ya algunos años, planteaba $T$. Nájera acerca de que algunas motillas comienzan a construirse en el Bronce Antiguo, caso de las del Azuer y Los Romeros o de las Morras de El Quintanar y El Acequión, y buena parte de estos monumentos se mantienen en uso hasta El Bronce Pleno Reciente (NAjERA, T. 1984, 23-24) por lo que este círculo cultural tiene un desarrollo sincrónico al Argar.

Una vida similar parecen tener algunos de los poblados situados en el reborde montañoso meridional y oriental como es el caso de La Encantada cuyos materiales están emparentados con el ámbito argárico, más que con el círculo de las motillas (CASTRO, P.; LuLL, V., y Micó, R., 1996, 127); o el poblado de las Hoyas del Castillo de Pajaroncillo, más ligado a los asentamientos de la cuenca del Tajo. Pero en ambos casos (motillas y poblados de altura) existen también ejemplos de ocupaciones mucho más cortas centradas en un momento más o menos avanzado de la Edad del Bronce, como lo demuestra el Cerro de El Cuchillo y, con menos datos, Las Motillas de Los Palacios y Santa María de Retamar (FernándeZ.POSSE, M.² D.; GlLMAN, A., y MARTín, C., 1996, 127-128).

Sin embargo, la escasa presencia de conjuntos Cogotas I conocidos en el sector suroriental de la submeseta sur al que se refiere el citado trabajo y la tradicional adscripción de este círculo dentro del Bronce Final explican que en dicha recopilación (FERNÁNDEZ-POSSE, M. ${ }^{2}$ D.; GILMAN, A., y MARTíN, C., 1996) se excluya expresamente este círculo cultural por estimarlo posterior al Bronce pleno, a pesar de que los datos que ofrece la submeseta norte empiezan a demostrar que sus inicios hay que remontarlos, al menos, 
a mediados del II milenio y, por tanto, resultan sincrónicos a algunos de los yacimientos a los que se hace referencia en el trabajo, como son Las Motillas o los poblados de altura. A pesar de esta expresa exclusión, algunos de las estaciones incluidas en el listado que se ofrece cuentan con niveles en los que se han recuperado materiales Cogotas I, como es el caso de Hoyas del Castillo (Pajaroncillo, Cuenca).

Estos pequeños avances en la definición cronológica de los círculos de la Edad del Bronce en el área suroriental de la Submeseta sur, no tienen paralelo en la cuenca del Tajo que apenas cuenta con dataciones absolutas, siendo especialmente escasas las referidas al círculo Cogotas I, uno de los Horizontes culturales de la Prehistoria reciente con más amplia difusión espacial dentro del territorio peninsular y, en particular, en la cuenca del Tajo. La carencia resulta más llamativa si la comparamos con el amplio repertorio de fechas de C14 con que cuenta ya la Prehistoria reciente peninsular (CASTRO, P.; LULL, V., y MICO, R., 1996) y justifica que, para definir el marco cronológico de esta área, constantemente haya que seguir recurriendo a los datos que han generado los yacimientos de la Edad del Bronce de la Meseta norte, a pesar de que tampoco en esta zona es muy larga la lista de dataciones radiocarbónicas.

La situación queda patente en una exhaustiva revisión cronológica del Horizonte Cogotas I para el conjunto de la Península realizada recientemente donde se evidencia esia carencia de dataciones en el Valle del Tajo y, especialmente, su total ausencia en los primeros estadios del horizonte haciéndose alusión al problema con las siguientes palabras: "Resulta extraño la inexistencia de yacimientos en el Valle del Tajo con fechas anteriores a c. $1400 \mathrm{cal}$ ANE, puesto que las relaciones Meseta Norte-Sudeste debieron ser vehiculizadas a través de aquel territorio. La referencia a yacimientos adscritos a la fase Proto-Cogotas I podría encaminarse a cubrir el intervalo de los siglos XVII-XV cal ANE en esta región" (CASTRO, P.; MiCo, R., y SANAhuja, E., 1995: 92).

Con el fin de comenzar a subsanar esta deficiencia de dataciones, tanto en lo que se refiere a la secuencia de la Edad del Bronce en el sector más septentrional de la Submeseta sur, como en lo relativo a la periodización y posición temporal del círculo Cogotas I en sus momentos iniciales, hemos intentado la obtención de varias dataciones de C14 en el yacimiento del Caserío de Perales del Río, un asentamiento situado en la cuenca baja del Manzanares, dentro del término municipal de Getafe (Madrid), que ha proporcionado un magnífico ejemplo de hábitat del Horizonte Cogotas I, ocupado a lo largo de las dos fases conocidas: Protocogotas I o Cogeces y Cogotas I de plenitud. Desgraciadamente, sólo una de las muestras enviadas al laboratorio ha permitido obtener un resultado positivo debido al fuerte proceso de mineralización que sufre la materia orgánica en el tipo de suelos en que se ubica el asentamiento. Este problema se contrarresta por el hecho de que el dato corresponde a una de "las fosas" de la fase inicial o Protocogotas I conviertiéndose en la primera datación que tenemos para esta facies y en esta área, además, cuenta con la ventaja de haberse obtenido a partir de una muestra de hueso procedente de una unidad cerrada (Fondo 3 de la cuadrícula 13) perfectamente contextualizada. A estas ventajas se suma el que la datación resultante se ajusta perfectamente a los valores que ofrecen otros análisis procedentes de yacimientos de la Meseta norte con contextos similares. 


\section{EL YACIMIENTO DE PERALES DEL RIO: UN MODELO DE ASENTAMIENTO COGOTAS I EN TERRAZA FLUVIAL}

El yacimiento fue excavado bajo la dirección de dos de nosotras (J. Calle y M. ${ }^{a} \mathrm{~L}$. Sánchez Capilla) y se encuentra ubicado dentro del término municipal de Getafe (Madrid), en la cuenca baja del Río Manzanares, en un paraje de especial concentración de yacimientos arqueológicos. Se trata de un clásico asentamiento de "fondos" de una gran extensión, del cual ya se han dado a conocer algunos aspectos puntuales, como sus enterramientos (BLASCO, C.; SÁnCHEZ-CAPILLA, L.; CALle, J.; ROBLES, F.; GONŹ́lez, V. M., y GONZÁLEZ, A., 1991). En él los materiales se concentran dentro de los hoyos o "fondos", en conjuntos cerrados, donde se aíslan perfectamente los restos correspondientes a cada una de las ocupaciones, por lo que nos encontramos ante un marco especialmente idóneo para definir las características y elementos de cada horizonte y asignar dataciones a cada una de las fases con cierta fiabilidad.

Por otra parte, la colmatación de las distintas "fosas" con materiales pertenecientes a, al menos, las dos fases del Horizonte Cogotas I, confirman que el lugar fue ocupado a lo largo de un período temporal relativamente prolongado, a pesar de no haberse formado, no sólo una estratigrafía , sino tampoco un suelo de ocupación más o menos somero. Un dato que habrá que tener en cuenta a la hora de hacer lectura de ciertas estratigrafias ya que, en este caso, de no haberse recuperado los materiales en unidades cerradas, hubiera sido imposible la diferenciación secuencial. Esta circunstancia explica la dificultad que muchas veces existe para definir la periodización de Cogotas I y, en consecuencia, para adscribir los materiales a una u otra etapa.

A modo de síntesis queremos apuntar que, además de las diferencias en la ornamentación cerámica más o menos establecidas a partir de los materiales recuperados en diversos yacimientos, existen otros elementos que definen, al menos, dos estadios. En primer lugar, las formas de las cerámicas cuidadas presentan carenas más acusadas y fondos netamente convexos en la etapa de formación, frente a los galbos más redondeados y las formas troncocónicas de la parte inferior de los recipientes de la etapa de plenitud. Otro dato diferenciador lo constituye la metalurgia, bastante arcaizante en la primera etapa y de influencia atlántica en la segunda, no sólo por la tipología de los objetos sino por la propia composición de los mismos, ya que, en los momentos avanzados del horizonte, es frecuente la presencia de bronces ternarios. Así mismo, las fíbulas de codo asociadas a contextos Cogotas I corresponden a las etapas epigonales del Horizonte. Por último, queda mencionar el cambio operado en la industria lítica, todavía con frecuente utilización de matrices laminares en la primera etapa y prácticamente reducida a algunos elementos de hoz sobre lascas relativamente espesas en la segunda.

En otro orden de cosas, las manifestaciones funerarias son más frecuentes en los primeros momentos y muestran estrechos paralelos con las propias de otros grupos del Bronce pleno de las tierras del interior peninsular, además de presentar algunas similitudes con la cultura argárica, como es la tendencia a la inhumación individual, la localización de las tumbas en el área de habitación o la posición contraída de los cuerpos. Por 
el contrario, en la etapa de plenitud dichas manifestaciones se enrarecen considerablemente aunque mantienen características similares.

En lo que se refiere a la fosa datada, tan sólo entregó algunos huesos animales y un conjunto de fragmentos cerámicos del que hemos seleccionado las piezas de las figuras 1 y 2. En este material cerámico se resume perfectamente las características formales y ornamentales, tanto de los recipientes comunes como de los cuidados, pertenecientes a la etapa Protocogotas I. En las cerámicas comunes, a veces correspondientes a recipientes de gran tamaño, la omamentación, si existe, se reduce a algunas aplicaciones plásticas de mamelones o cordones lisos o impresos, como muestran los ejemplares 1 y 2 de la figura 1. En cuanto a las series cuidadas, destacan las carenas más o menos acusadas y las decoraciones de incisiones o impresiones en zig-zag o espigas asi como los puntillados relativamente gruesos sin combinar con incisiones u otras técnicas (figura 2.3, 2.4 y 2.5).

Además, entre los materiales obtenidos en "fondos " próximos al que ha sido datado destacan, junto a las cerámicas con características como las apuntadas, algunos instrumentos metálicos, recuperados en escasas proporciones, que ofrecen una tipologia propia de las producciones de los círculos del Bronce Medio, es el caso de un punal de remaches, una punta de pedúnculo prolongado y diversos punzones y varillas de sección cuadrangular, todos ellos de morfologia cercana a los instrumentos de producción argáricos (LUL, V., 1983, 214-219). Todos estos objetos han sido elaborados en una aleación de bronce binario con una presencia de estaño que oscila entre el 8 y el $20 \%$ (BLAS$\mathrm{CO}, \mathrm{C}$., y ROVIRA, S., 1992-93, 411), la excepción la constituye la única punta recuperada que ha sido realizada en un cobre con algunas impurezas.

Completan el equipo material recuperado en los fondos próximos al datado algunos elementos líticos que, en muchas ocasiones, están elaborados todavía sobre láminas, como sucede en el caso de las motillas (NÁJERA, T.; MOLINA, F.; TORRE DE LA, F.; AGUAYO, P., y SÁEZ, L., 1979, 34, fig. 7,i) o en otros yacimientos de diversa indole, pertenecientes a la Edad del Bronce Antiguo y Medio de la Submeseta Sur (BuRGAleta, J., y SÁnCHEZ MESEGUER, J. L., 1988, 291-300). Así mismo, entre el material pulimentado y pesado cabe mencionar algunas hachas y azuelas pulimentadas y, por su abundancia, los molinos barquiformes, realizados con granito ajeno a la litologia del entorno.

Por todo ello, tanto la cerámica como las industrias lítica y metálica vinculan, sin ningún género de dudas, esta fosa y un gran conjunto de fosas adyacentes a la etapa Protocogotas I, cuyos materiales coinciden con los entregados por un importante número de estaciones de la Meseta norte, muchas de ellas, como veremos, con dataciones que se aproximan a la deparada por el Caserio de Perales del Río.

\section{LA DATACIÓN DE C14 DE PERAIES DEL Rfo EN EL CONTEXTO CRONOLÓGICo DE LA ETAPA PROTOCOGOTAS I}

Como ya se ha apuntado, la fecha fue obtenida a partir de hueso animal recuperado en la fosa 3 de la cuadrícula 13 y se procesó en el Instituto Rocasolano del CSIC, gra- 


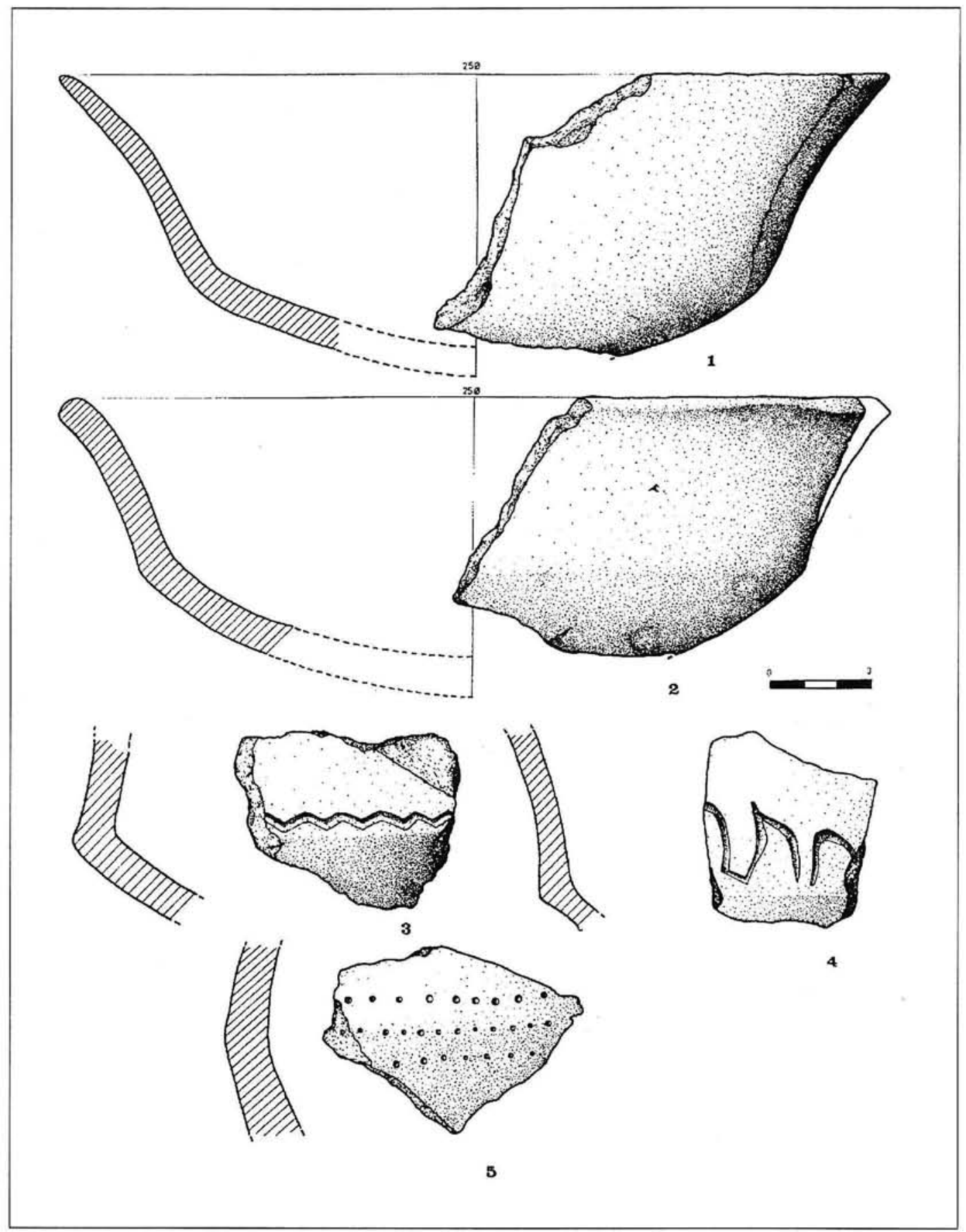

Figura 1. Recipientes cerámicos recuperados en la fosa datada. 1.1 y 1.2 Grandes vasijas de almacenaje decoradas, respectivamente, con cordón liso e impresiones. 1.3 Cuenco hemiesférico liso 


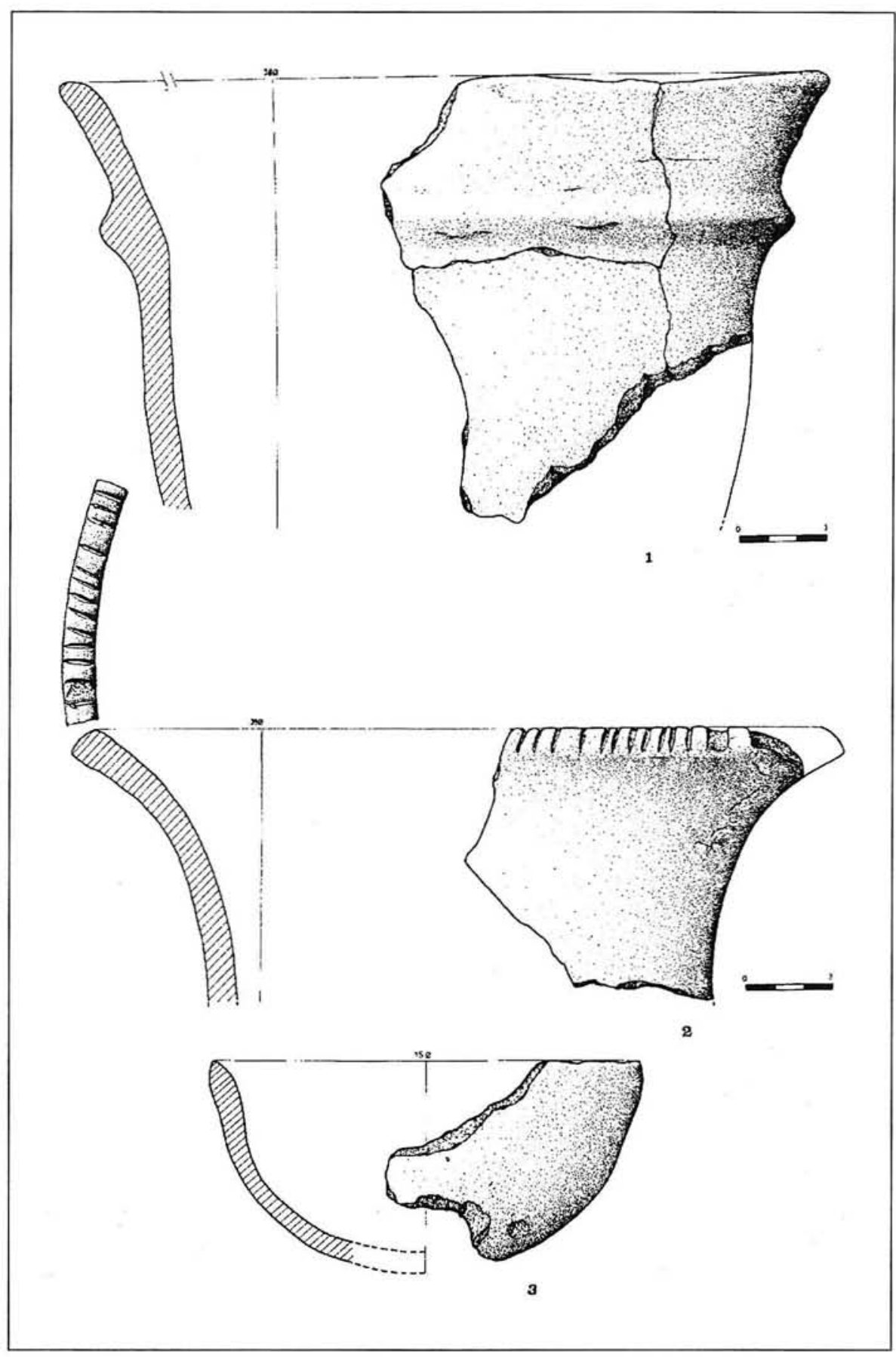

Figura 2. Recipientes cerámicos recuperados en la fosa datada. 2.1 y 2.2 cazuelas lisas de superficies cuidadas. 2.3, 2.4 y 2.5 fragmentos decorados 
cias a la amable colaboración del Dr. Fernán Alonso, a quien desde aquí agradecemos su contribución. El resultado fue el siguiente:

\begin{tabular}{|l|l|l|l|l|l|l|}
\hline $\begin{array}{l}\text { situación } \\
\text { muestra }\end{array}$ & Laboratorio & bp & ane ac & \pm ane & $\begin{array}{l}\text { cal. Pearson y Stuiver } \\
(1993) \mathrm{BC}\end{array}$ & Muestra \\
\hline $\begin{array}{l}\text { fondo 3 } \\
\text { cuad.13 }\end{array}$ & CSIC- 1089 & 3356 & 1406 & 68 & 1629 & Hueso \\
\hline
\end{tabular}

Aunque los materiales cerámicos que pueden incluirse en la fase Protocogotas I son muchos, pocas veces se encuentran en contextos claros que permiten una adscripción segura y, por tanto, son pocas las fechas conocidas que facilitan un término de comparación para la datación que damos a conocer. Concretamente en la Submeseta Sur el único dato de comparación cronológica que poseemos nos lo ofrece el yacimiento de Hoyas del Castillo (Pajaroncillo, Cuenca), situado en la cuenca alta del rio Cabriel, en el entorno de la Serranía de Cuenca y, por tanto, fuera de la cuenca del Tajo donde se ubica el Caserio de Perales. Dicho yacimiento ha proporcionado una importante secuencia que abarca todo el II milenio a. C., entre su material cerámico son característicos los perfiles carenados asi como determinados motivos y técnicas ornamentales (ULREICH, H.; NEGRETE, M." A., y PUCH, E., 1994, 131). En dicha secuencia el estrato 6 nos ofrece un contexto que podría encuadrarse dentro de la fase Protocogotas I aunque algo avanzada por la presencia de un fragmento con decoración de boquique (p. 120 , fig. 6, n. $^{\circ} 8$ a 17) este estrato ha ofrecido las siguientes dataciones:

$$
\begin{array}{llll}
\text { P-4072-A } & \text { B-5417 } & 3250+70 \text { b.p. }= & 1300 \text { B.C. } 1540 \mathrm{cal} \mathrm{BC} \\
\text { P-4069-A } & \text { B-5418 } & 3200+70 \text { b.p. }= & 1250 \text { B.C. } 1485 \text { cal BC }
\end{array}
$$

La mayor antigüedad de la datación del Caserío de Perales con respecto al nivel 6 de Hoyas del Castillo se explicaría por la ausencia total de elementos omamentales teóricamente recientes, como es el caso de las técnicas de boquique y excisión, una ausencia que también caracteriza a algunos conjuntos de la Meseta Norte que han proporcionado dataciones que permiten una contrastación más precisa con el yacimiento madrileño.

Uno de los yacimientos culturalmente más próximos al Caserio de Perales es el de Los Tolmos de Caracena, esta cercanía entre ambos yacimientos se certifica también en su cronología ya que las dataciones del yacimiento soriano son las siguientes:

$$
\begin{aligned}
& \text { C.S.I.C. } 409=3.360+50 \text { b.p. }=1410 \text { a.C. } 1668 \text { cal BC } \\
& \text { C.S.I.C. } 408=3.370+50 \text { b.p. }=1420 \text { a.C. } 1676 \text { cal BC } \\
& \text { C.S.I.C. } 407=\quad 3.010+50 \text { b.p. }=1060 \text { a.C. } 1295 \text { cal BC }
\end{aligned}
$$




$$
\begin{aligned}
& \text { C.S.I.C. } 480=3.380+50 \text { b.p. }=1430 \text { a.C.1682 cal BC } \\
& \text { C.S.I.C. } 443=3.360+50 \text { b.p. }=1410 \text { a.C. } 1668 \text { cal BC } \\
& \text { C.S.I.C. } 442=3.380+50 \text { b.p. }=1430 \text { a.C.1682 cal BC } \\
& \text { C.S.I.C: } 479=3.180+50 \text { b.p. }=1230 \text { a.C. } 1466 \mathrm{cal} \mathrm{BC}
\end{aligned}
$$

(JiMENo, A., 1982, 337-338; CASTRO, P.; LULL, V., y MiCo, R., 1996).

Como puede comprobarse, la datación del Caserio de Perales coincide exactamente con las proporcionadas por las muestras números 409 y 443 de Los Tolmos, ya que en la fecha del yacimiento madrileño no se ha redondeado a la decena más próxima. Esta coincidencia resulta significativa si tenemos en cuenta la similitud de materiales entre ambos yacimientos, tanto en lo que se refiere a las cerámicas, como a las características de la metalurgia, un paralelo que se acentúa si consideramos que en ambos conjuntos se documentaron hábitats asociados a enterramientos, así como sucesivas ocupaciones pertenecientes, tanto a la fase Protocogotas I como a la etapas Cogotas I de plenitud (Jimeno, A., y FernÁNDEZ MORENo, J. J., 1991). Precisamente esta reiterada ocupación de Los Tolmos explica la existencia de fechas algo más bajas, como son las que proporcionan las muestras 407 y 479.

El dato que ofrece Perales del Río se encuentra también avalado por otro yacimiento de características similares e idéntica adscripción cultural ocupado exclusivamente en la etapa Protocogotas I que ha sido estudiado y datado recientemente, nos estamos refiriendo al campo de fosas de "El Cogote" (Avila) que ha entregado dos fechas obtenidas en las fosas 11 y 4 respectivamente, con los siguientes valores:

$$
\begin{aligned}
& \text { Fosa 11: Gr N } 18873 \ldots . .3 .330 \div 35 \text { b.p. }=1385+35 \text { a.C. } 1650 \mathrm{cal} \mathrm{BC} \\
& \text { Fosa 4: Gr N } 18874 \ldots . .3 .415+40 \text { b.p. }=1465+40 \text { a.C. } 1716 \mathrm{cal} \mathrm{BC}
\end{aligned}
$$

(Caballero, J.; Porres, F., y Salazar, A., 1993, 106, y Fabián, F. J., 1993, 165; Castro, P.; LULL, V., y MiCO, R., 1996).

Un panorama cronológico muy similar nos ha ofrecido el yacimiento salmantino de La Corvera, considerado igualmente como un ejemplo típico de la etapa Protocogotas I y del que se conocen dos fechas; la segunda de las cuales, como en el caso de Los Tolmos, resulta también absolutamente coincidente la del Caserio de Perales:

$$
\begin{aligned}
& 3315+25 \text { bp } 1365+25 \text { a.C. } 1587 \mathrm{cal} \mathrm{BC} \mathrm{y} \\
& 3355+25 \text { bp } 1405+25 \text { a.C. } 1655 \mathrm{cal} \mathrm{BC}
\end{aligned}
$$

(FABIÁ, F. J., 1993, 165; CASTRO, P.; LULL, V., y MiCO, R., 1996).

Una lectura algo distinta podemos hacer de las dataciones obtenidas en el yacimiento palentino de la Venta, cuyos materiales parecen centrar también su ocupación 
en la fase Protocogotas I. La diferencia estriba en que las dos dataciones obtenidas sobre carbones de una misma fosa (65-E) han entregado resultados algo dispares:

$$
\begin{aligned}
& 3100+50 \text { bp } 1150+50 \text { a.C. } 1382+50 \mathrm{cal} \mathrm{BC} \mathrm{y} \\
& 3300+35 \text { bp } 1350+35 \text { a.C. } 1583+35 \text { cal BC }
\end{aligned}
$$

(Pérez Rodríguez, F. J., y fernández Giménez, J. M., 1993, 41; Castro, P.; lull, V., y MiCO, P., 1996).

En efecto, mientras la primera de estas fechas resulta excesivamente reciente, a pesar de corresponder al relleno más moderno, la segunda, procedente del lecho de la base de la fosa, es prácticamente idéntica a las obtenidas en El Cogote o La Corvera y muy próxima a algunas de Los Tolmos y al propio Caserío de Perales. El distanciamiento entre ambas dataciones sólo se justificaria por un prolongado proceso de relleno de la fosa, pero desconocemos las características concretas de los materiales arqueológicos depositados en esta unidad, asi como el contenido geológico de su relleno.

Otros datos que nos permiten comparaciones precisas nos los ofrece el yacimiento de La Plaza (Cogeces del Monte), el primero en el que se aisló con nitidez la fase Protocogotas I (DeLIBES, G., y FERNÁNDEz MANZANO, J., 1981), y del que posteriormente se han conocido dos dataciones obtenidas a partir de madera carbonizada recuperada bajo los derrumbes de la muralla, las cuales vuelven a situar la etapa Protocogotas I no lejos del marco temporal al que apunta la fecha del Caserío de Perales del Río, estas dataciones son:

$$
\begin{aligned}
& \text { Gr N } 10.617=3275+30 \text { bp } 1325+30 \text { a.C.1560 + } 30 \text { cal BC } \\
& \text { Gr N } 14.560=3275+\text { ? bp } 1325+\text { ? a.C. } 1560+\text { ? Cal BC }
\end{aligned}
$$

(Rodriguez Marcos, J. A., y abarquero, F. J., 1994: 52; Castro, P.; Lull, V., y Mico, R., 1996).

Algo alejada del resultado del Caserío de Perales del Río se encuentran, en cambio, la datación obtenida en La Huelga y que corresponden a las fosas H-171 de la Huelga 1 y AF 226 de La Huelga 2, son las siguientes:

$$
\begin{aligned}
& \text { Beta } 71373=3080+60 \text { b.p. } 1130+60 \text { a.C. } \\
& \text { Beta } 71374=3160+60 \text { b.p. } 1210+60 \text { a.C. }
\end{aligned}
$$

(Perez Rodrfguez, F.; Misiego, J. C.; Sanz, F.; Marcos, G.; Carbajo, M. A., y Fernández, J. M., 1994, 26).

El distanciamiento es acusado si se tiene en cuenta que el valor que nos interesa es el primero pues, según sus excavadores, el sector 2, al que se refiere la segunda fecha, 
ha deparado fundamentalmente materiales pertenecientes al Horizonte campaniforme tipo Ciempozuelos, mientras que el sector 1 corresponde a un asentamiento netamente Protocogotas I (PÉRez Rodríguez, F; Misiego, J. C.; SANZ, F.; Marcos, G.; CARBajo, M. A., y FERNÁNDEZ, J. M., 1994, 24) y, por tanto, es esta área la que se encuentra directamente vinculada desde el punto de vista cultural y cronológico al Caserío de Perales del Río. Esta circunstancia hace muy difícil la lógica de la datación, pues resulta más moderna la fecha del horizonte teónicamente más antiguo.

Otro es el caso de las dataciones, también problemáticas, que nos han ofrecido algunos de los yacimientos en cueva del sector oriental de la Meseta norte con niveles pertenecientes al Horizonte Cogotas I y, más concretamente, a la facies Protocogotas I, como ejemplo hay que citar la Cueva de la Vaquera (Torreiglesias, Segovia), donde la secuencia no parece ajustarse a la periodización tradicional ya que los materiales Cogotas I clásicos aparecen infrapuestos a los correspondientes a la fase Protocogotas I, los cuales ofrecen una datación de:

$$
\text { C.S.I.C. }-340 \quad 3380+50 \text { b.p. } \quad 1430+50 \text { a.C. } 1682+\text { cal BC }
$$

(EIROA, J., 1979, 69; CASTRO, P.; LULL, V., Y MICO, R. 1996).

Igualmente compleja resulta la interpretaciốn de La Cueva del Asno (Los Rábanos, Soria), donde existen materiales cerámicos que podrían pertenecer a la fase Protocogotas I (Eiroa, J., 1979, n. ${ }^{\circ} 14,17,33,35$ del inventario) pero ninguna de estas cerámicas se recuperaron en el nivel y sector donde se obtuvo una de las dataciones que encajaría perfectamente con este horizonte cultural:

$$
\text { C.S.I.C.-340 } 3380 \div 50 \text { b.p. } \quad 1430+50 \text { a.C. } 1682+\text { cal BC }
$$

(EIROA, J., 1979, 69; CASTRO, P.; LuLL, V., y MiCo, R. 1990).

Una casuística distinta ofrece la Cueva de Arevalillo de Cega, cuyo nivel IIa entregó también una serie de materiales adscribibles a la fase Protocogotas I, aunque asociados tanto a elementos propios de Cogotas de plenitud como al Horizonte campaniforme, algo que fue interpretado como la evidencia del surgimiento de Cogotas I como derivación directa del campaniforme, pero también permitió confirmar la temprana for" mación del Horizonte Cogotas I dentro del II milenio a.C., en momentos sincrónicos al desarrollo del Bronce Medio (FerNÁNDEZ POSSE, M. ${ }^{2}$ D., 1981: 76- 81). Sin embargo, estas asociaciones deben de ser manejadas con cuidado ya que podríamos encontrarnos ante un nivel con materiales procedentes de ocupaciones distintas que no legaron a formar acumulaciones estratigräficas y, en consecuencia, no es posible diferenciar los elementos de cada uno de los asentamientos. De todas formas, este nivel Ila ha proporcionado tres dataciones, todas bastante acordes con las más habituales de la Fase Protocogotas I, son las siguientes: 
C.S.I.C. $-400-\quad 3290+50$ b.p. $\quad 1340+50$ a.C. $1576+50$ cal BC

C.S.I.C. -422 y $423=3300+50$ b.p. $\quad 1350+50$ a.C. $1581+50$ cal BC

(FERnÁNDEZ POSSE, M. D., 1981, 51; CASTRO, P.; Lull, V., y Mico, R., 1996).

La nómina de yacimientos Protocogotas I no se cierra con los conjuntos citados sino que se amplia día a día, tanto con conjuntos que sólo tienen ocupación en esta fase como con otros de cronología más amplia que puede abarcar todo el Horizonte, comc es el caso del Caserío de Perales del Río, pero de momento no contamos con más data. ciones radiocarbónicas que sirvan de término de comparación. No obstante, y aun de jando aparte las dataciones de contexto inseguro o de lectura problemática, los datos con los que contamos son lo suficientemente coherentes como para afirmar que la primera etapa del Horizonte Cogotas I, identificada como Cogeces o Protocogotas I se centra entre los siglos XV y XIV A. C., en fechas no calibradas, unos valores que entran perfectamente dentro del margen de 1800-1550 cal ANE., en el que algunos autores har centrado la Fase I de Horizonte Cogotas I (Castro, P, V. Lull y R. Micó, 161).

\section{LA SINCRONIA Y PROXIMIDAD CULTURAL DE LA FASE PROTOCOGOTAS I CON OTROS CÍRCULOS DEL BRONCE PLENO PENINSULAR:}

Con estos límites temporales, parece evidente que la etapa Protocogotas I se desarrolla en paralelo a otros círculos peninsulares del Bronce Pleno como son el Argar, e Bronce Valenciano o el Bronce de las Motillas, pero también es sincrónica a la vida de algunos poblados ubicados en las cuencas sedimentarias del Duero y Tajo, en parajes y con caracteristicas y equipos materiales similares a la mayoria de los hábitats cogotas ] más tempranos, aunque sin las específicas cerámicas con decoraciones de incrustación El ejemplo más significativo de este tipo de yacimientos es el de La Loma del Lomo (Co golludo, Guadalajara), con una prolongada ocupación a lo largo de un milenio (desde mediados del III milenio, hasta avanzado el II milenio a. C.), el cual ha proporcionadc un total de seis dataciones radiocarbónicas de las que, las dos más recientes, realizadas a partir de restos recuperados en sendas fosas de enterramiento, han proporcionado una cronología coincidente con los momentos iniciales de Cogotas I, estos valores son:

El Lomo n. ${ }^{\circ}$ 3: $1-14.220 \quad 3450+160$ b.p. $=1500+160$ a. C.

(VALIENTE, J., 1987, 159).

El Lomo n. ${ }^{\circ}$ 4: I-14.891 $3340+100$ b.p. $=1390+100$ a.C. $1630+100$ cal BC (Valiente, J., 1992, 195; CAstro, P.; LuLl, V., y Mico, R., 1996).

La coincidencia de estas fechas con las que hemos venido analizando resulta más llamativa si tenemos en cuenta que, además de las similitudes materiales de este yaci- 
miento con los contextos Protocogotas I, existen también estrechos paralelos en las características de los enterramientos y en los ritos funerarios en general, así como en el tipo de hábitats y algunos de sus elementos muebles. Esta sincronía entre grupos con cerámicas lisas y la etapa inicial de Cogotas I confirma que este círculo cultural pudo originarse en el seno de los grupos meseteños del Bronce Pleno, sin que en los primeros momentos de su desarrollo se adviertan grandes diferencias entre quienes adoptan un nuevo gusto decorativo en un pequeño porcentaje de recipientes cerámicos, empleando técnicas de incrustación, como la incisión la impresión o el puntillado, y quienes mantienen la tradición de dejar las superficies lisas en sus recipientes más cuidados, por lo que la diferencia más llamativa entre unas comunidades y otras es precisamente esta peculiar forma de ornamentar, con temas muy simples y repetitivos.

Pero la proximidad cultural de Protocogotas I no sólo se observa con grupos asentados en las cuencas sedimentarias de los rios Duero y Tajo, con un modelo de hábitat muy parecido, sino que también se produce con otros círculos del Bronce Pleno peninsular que, aunque con asentamientos de características distintas y diferentes estrategias de control de recursos, comparten tecnologías cerámica, metalúrgica o lítica y muestran semejanzas en otros aspectos como sus costumbres funerarias, estos paralelos se explican en el marco de unas relaciones fluidas y recíprocas donde es difícil determinar los préstamos de unos y otros, pero dentro del cual se entiende la gran difusión que alcanza el estilo cerámico de las gentes Cogotas I, bien por comercio o, como parece más frecuente, por imitación de unos modelos conocidos a consecuencia de contactos originados por causas muy diversas.

Otra cuestión no menos debatida es la sincronía o diacronia del fenómeno Cogotas I entre la Meseta y las áreas periféricas pues, aunque el mayor número de yacimientos correspondientes a la etapa Protocogotas se concentra en la Meseta Norte y en el Valle del Tajo, no se puede olvidar que son ya muchas las estaciones periféricas que han entregado materiales que se pueden adscribir a esta misma fase, posiblemente con una antigüedad similar a los yacimientos de la Meseta, aunque, desgraciadamente, apenas contamos con dataciones absolutas que garanticen esta sincronía, bien porque proceden de contextos poco claros, bien porque no parecen estar en armonía con el tipo de materiales al que se asocian. En este sentido, hay que mencionar los problemas que las fechas de Moncín o de Fuente Álamo presentan para los propios investigadores de los respectivos yacimientos (SCHUJART, H., y ARTEAGA, O.,1983 y 1986, y HARRISON, R. J.; MORENO, G., y LEGGE, A. J., 1994). Por ello, de momento, los únicos datos que pueden valorarse proceden de dos yacimientos andaluces: Peñalosa y Setefilla, con cronologias bastante antiguas que confirman una posición temporal de los materiales Protocogotas similar a la de los conjuntos meseteños de este Horizonte. Muchas más dudas para ser clasificado como Protocogotas I presenta el yacimiento de Gatas, con fechas algo más recientes.

Concretamente, en los estratos XV y XIV de la Mesa de Setefilla se recuperaron algunos ejemplares cerámicos que podrían encuadrarse en esta primera fase de Cogotas I y cuyo momento final ha proporcionado una datación que fue considerada excesivamente alta por algunos autores, aunque hoy ya no está tan distante de los parámetros 
Protocogotas I de la Meseta, por lo que podría aceptarse, aunque requeriria una contrastación, la datación es la siguiente:

I-11.070 Setefilla 5 …. $3.520+95$ B. P. $1570+95$ a. C. $1859+95$ cal BC (Aubet, M. ${ }^{2}$ E.; Serna, M. ${ }^{2}$ R.; Escacena, J. L. , y Ruiz, M., 1983, 48; CaSTro, P.; LuLl, V., y MICO, R., 1996).

El segundo yacimiento con cerámicas netamente Protocogotas I es Peñalosa, donde "dichas cerámicas formaban parte de los ajuares domésticos del último momento del asentamiento argárico. Todas las dataciones de este asentamiento se han obtenido a partir de muestras de vida larga, vigas y postes de las estructuras arquitectónicas, de manera que las cerámicas decoradas deben ubicarse con posterioridad a las fechas más recientes que se sirúan c. 1750-1700 cal ANE" (CASTRO, P.; MiCO, R., y SANHAujA, E., 1995, 90), estas "cerámicas decoradas... como imitaciones de los estilos típicos de Cogotas indicanl que ya en esta época, a partir del 1400 , se establecieran contactos entre las poblaciones ganaderas de la Meseta y las del Alto Guadalquivir" (CONTRERAS, F., 1995: 147). Lo cierto es que estas dataciones encajan perfectamente con los valores obtenidos en yacimientos mesetenos con materiales de similares características.

Más dudas nos plantea la adscripción de los materiales de Gatas a la etapa ProtoCogotas I pues, según se afirma, incluye "cerámica con decoración de boquique" (CASTRO, P.; MiCO, R., y SANHAUIA, E., 1995, 91), lo que permite suponer que corresponde a un momento algo más avanzado, en el que encajaría la datación obtenida:

\section{c. $1550 / 1525$ cal ANE}

(CASTRO, P.; Mico, R., y Sanhauja, E., 1995, 91).

El resto de los yacimientos periféricos a la Meseta con materiales Protocogotas I no han ofrecido dataciones imputables, con seguridad, al contexto en que se recuperaron, no obstante, si conocemos los conjuntos a los que se asocian y todo hace suponer que son sincrónicos a desarrollos culturales bien definidos y datables a mediados del II milenio, como puede deducirse del poblado de La Horna (Aspe, Alicante) en el Valle medio del Vinalopó, que no ha sido fechado, aunque el contexto general permite encontrar los "paralelos más próximos en el poblado argárico de El Picacho con dataciones del 15001440 a. C." (HERNÁNDEZ, M., y DUG, I., 1994, 93).Estos ejemplos no son más que la muestra de una larga lista de yacimientos del entorno de la periferia de la meseteña, en puntos del Valle del Ebro, Levante, cuenca del Guadalquivir, etc, que han entregado materiales protocogotas más o menos abundantes asociados a contextos de diversos círculos del Bronce pleno aunque, a veces, la rara formación de suelos de ocupación, consecuencia de los frecuentes establecimientos temporales, impide aislarlos de otras ocupaciones anteriores o posteriores de la edad del Bronce y, por tanto, darles una adscripción temporal precisa. 
Ante todos estos datos, muy fragmentarios todavía, debemos de mantener cierta prudencia sobre los posibles focos de origen del Horizonte Cogotas I, ya que son necesarios más argumentos que los disponibles en la actualidad para poder pronunciarse, no obstante, podemos afirmar que dicho Horizonte debió alcanzar, desde sus origenes, una amplia extensión en la Meseta y fuera de ella dentro de un marco general de fluídos intercambios y relaciones promovidos posiblemente por distintos factores, entre los que no se descartan las trashumancias ganaderas, tal como se ha apuntado reiteradamente, pero tampoco podemos olvidar, entre las causas de esta fluidez de relaciones, el comienzo "de la explotación intensiva de los recursos mineros del Piedemonte de Sierra Morena" (CONTRERAS; F., 1995, 146) un hecho que está avalado por dataciones de C14 a partir del 1500/1400 y que determina, entre otras causas, el establecimiento de una serie de poblados de nueva planta (Terrera del Reloj, Cerro del Alcázar, Rincón de Olvera y Penalosa) (CONTRERAS, F., 1995, 146), asi como la prospección y beneficio de otras zonas mineras $O$ intereses meramente mercantiles.

En suma, fecha del Caserío de Perales que damos a conocer confirma la sincronía del desarrollo del Horizonte Cogotas I en las cuencas del Duero y del Tajo y permite sospechar que es desde este segundo valle desde donde se produce la temprana expansión hacia el Levante y la cuenca del Guadalquivir, si bien queda un importante vacio que es La Mancha y, en general, el Valle del Guadiana, aunque también existen indicios cada vez más numerosos de su participación en este fenómeno expansivo de Cogotas 1 .

\section{BIBLIOGRAFIA}

AUbET, M. ${ }^{2}$ E.; SERNA, M. ${ }^{2}$ R.; ESCACENA, J. L., y RUIZ, M. (1983): La Mesa de Setefilla. Lora del Río (Sevilla). Campaña de 1979. Excavaciones Arqueológicas en España, 122. Madrid.

BlASCO, C., y ROVIRA, S. (1992-93): "La metalurgia del cobre y del Bronce en la región de Madrid". Tabona VII, tomo II. La Laguna, 397-416.

Blasco, C.; Sánchez-Capilla, L.; Calle, J.; Robles, F; GonzÁlez, V. M., y GonzÁlez, A. (1991): "Enterramientos protocogotas en el Valle del Manzanares". Cuadernos de Prebistoria y Arqueologia de la UAM, 18. Madrid: 55-112.

Burgaleta, J., y SÁNCHEZ Meseguer, J. (1988): "Consideraciones en torno a la industria lítica de la Edad del Bronce en la Mancha". Actas del I congreso de Historia de Castilla-La Mancba, tomo II. Pueblos y culturas prebistóricas y protobistóricas (1). Junta de comunidades de Castilla-La Mancha. Ciudad Real: 291-300.

Caballero, J.; Porres, F., y Salazar, A. (1993): "El campo de fosas de "El Cogote" (La Torre, Ávila)". Numantia, 4. Valladolid: 93-110.

CASTRO, P.; LuLL, V., y MICO, R. (1996): Cronología de la Prebistoria Reciente de la Península lbérica y Baleares (c. 2800-900 cal ANE). BAR, Internnational Series 652. Oxford. 
CASTRO, P.; Mico, R., y SANahuja, E. (1995): "Genealogía Cronología de la "Cultura Cogotas I". (El estilo cerámico y el grupo de Cogotas I en su contexto arqueológico)". Boletín del Seminario de Arte y Arqueología, LXI, Valladolid, 51-118.

CONTRERAS, F. (1995): "Peñalosa. Un proyecto de investigación de la Edad del Bronce en el Alto Guadalquivir". Trabalbos de Antropología e Etnología. Vol. 35 (I). Porto: 143-158.

Delibes, G., y Fernández Manzano, J. (1981): "El Castro protohistórico de La Plaza en Cogeces del Monte (Valladolid). Reflexiones sobre el origen de la fase Cogotas $\mathrm{I}^{\mathrm{t}}$. Boletin del Seminarios de Arte y Arqueologia XLVI Valladolid: 51-68.

EIROA, J. (1979): La cueva del Asno. Los Rábanos (Soria), campañas 1976-1977. Excavaciones Arqueológicas en España, 107. Madrid.

FABí́N, J. F. (1993): "La secuencia cultural durante la Prehistoria reciente en el sur de la Meseta Norte". Trabalbos de Antropologia e Etnologia Vol. 33 (1-2). Porto: 145-178.

FERNÁNDEZ-POSSE, M. ${ }^{2}$ D. (1981): "La Cueva de Arevalillo de Cega (Segovia)". Noticiario Arqueológico Hispánico, 12. Madrid: 43-84.

FERNÁNDEZ-POSSE, M. ${ }^{2}$ D.; GILMAN, A., y MARTIN, C. (1996): "Consideraciones cronológicas sobre la Edad del Bronce en la Mancha". Homenaje al Profesor Manuel FernándezMiranda. II. Complutum extra 6.II. Madrid, 111-137.

Harrison, R.; MORENo, G., y LegGe, A. J. (1994): Moncin: Un poblado de la Edad del Bronce (Borja, Zaragoza). Zaragoza.

HERNÁNDEZ, M., y DUG, I. (1994): "La Homa (Aspe, Alocante), un yacimiento de la Edad del Bronce en el medio Vinalopó". Archivo de Prebistoria Levantina, XXI, Valencia, 83-118.

JIMENO, A. (1982): "Las fechas C14 del yacimento de Los Tolmos de Caracena (Soria)". Trabajos de Prebistoria, 39. Madrid 335-341.

- (1984): Los Tolmos de Caracena (Soria). (Campañas de 1977, 1978 y 1979). Nuevas bases para el estudio de la Edad del Bronce en la zona del Alto Duero. Excavaciones Arqueológicas en España 134. Madrid.

JIMENO, A., y FERNÁNDEZ MORENO, J. J. (1991): Los Tolmos de Caracena (Soria), (Campañas 1981 y 1982). Aportación al Bronce Medio en la Meseta. Excavaciones Arqueológi cas en España 161. Madrid.

Lull, V. (1983): La "Cultura" de El Argar Madrid.

MARTINEZ NAVARRETE, M." I. (1988): "Morras, motillas y castillejos zunidad o pluralidad cultural durante la Edad del Bronce en la Mancha". Homenaje a Samuel de los Santos. Albacete, 81-92.

NÁJERA, T. (1984): La edad del Bronce en la Mancha occidental. Tesis doctorales de la Universidad de Granada 458. 
NÁfeRA, T.; MOLINA, F.; TORRE DE LA, F.; AGUADO, P., y SÁ̇Z, L. (1979): "La motilla del Azuer (Daimiel; Ciudad Real). Campaña de 1976". Noticiario arqueológico bispánico, 6. Madrid, 21-50.

PEARSON, G.W., y Stuiver, M. (1993): Radiocarbon, 35, 25-33.

PéreZ RODŔgueZ, F. J., y FERNÁNDEZ GiméneZ, J. M." (1993): "Sobre la cocción de cerámica durante la Edad del Bronce. El yacimiento de "La Venta" (Alar del Rey, Palencia)". Numantia 4. Valadolid: 47-60.

Pérez Rodriguez, F. J.; Misiego, J.A.; Sanz, F. J.; Marcos, G. J.; Martín, M. A., y FernánDEZ, J. M. ${ }^{2}$ (1994): "La Huelga". Un interesante yacimiento de la Edad del Bronce en el centro de la Cuenca del Duero (Dueñas, Palencias)". Numantia, 5. Valladolid: 11-57.

RODRigueZ, J. A., y ABARQUERO, F. J. (1994): "Intervención arqueológica en el yacimiento de la Edad del Bronce de "El Cementerio- El Prado", Quintanilla de Onésimo (Valladolid)". Numantia 5. Valladolid; 33-57.

SCHUBART, H., y ARTEAGA, O. (1983): "Excavaciones en Fuente Álamo. La Cultura de El Argar". Revista de Arqueología 24, 25 y 26.

SCHUBART, H., y ARTEAGA, O. (1986): "Fundamentos arqueológicos para el estudio socioeconómico y cultural del área de El Argar". Homenjae a Luis Siret (1934-1984). Sevilla, pp. 289- 307.

Ulreich, H.; Negrete, M." A., y Puch, E. (1994): "Cerámica decorada de Hoyas del Castillo (Pajaroncillo, Cuenca) Corte 4". Boletin del Seminario se Arte y Arqueología, LX.: 105-135.

VALIENTE, J. (1987): La Loma del Lomo I. Cogolludo, Guadalajara. Excavaciones arqueológicas en España 152. Madrid.

- (1992): La Loma del Lomo II. Cogolludo (Guadalajara). Junta de Castilla-La Mancha. Toledo.

ZAMORA, A. (1976): Excavaciones de la cueva de La Vaquera. Torreiglesias, Segovia (Edad del Bronce). Segovia. 